\title{
Thiotepa versus Bacille Calmette-Guérin in Non-Muscle Invasive Bladder Cancer
}

\author{
Fatemeh Fallah $^{\mathrm{a}} \quad$ Mahdi Fallah $^{\mathrm{b}} \quad$ Raheleh Sadat Sajadi Nia ${ }^{\mathrm{a}}$

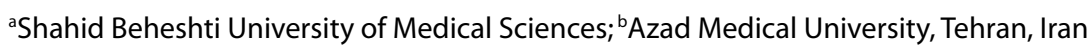

\section{Key Words}

Bacille Calmette-Guérin • Intravesical administration • Non-muscle invasive bladder cancer • Thiotepa

\begin{abstract}
Objective: The efficacy of intravesical thiotepa was evaluated compared with administration of Bacille Calmette-Guérin (BCG) in non-muscle invasive bladder cancer. Patients: In this multicenter, prospective, randomized study, eligible patients were those with proven non-muscle invasive bladder cancer. All patients were randomly allocated to Group A, receiving intravesical thiotepa (at a dose of $30 \mathrm{mg} / 30 \mathrm{ml}$ ) once weekly for 9 consecutive weeks and then monthly for 12 months or Group B, receiving intravesical Bacille Calmette-Guérin (Connaught strain, $80 \mathrm{mg} / 50 \mathrm{ml}$ ) over a 9-week induction course and each week for 3 weeks at 3, 6 and 12 months. Outcome measures were recurrence rate, time to first recurrence and progression rate. Treatment-related complications were also evaluated. Results: Seventy-two participants were enrolled, 36 for each group, 17 in Group A developed disease recurrence versus 25 of those in Group B $(p<0.05)$. There was no statistically significant difference in mean time to the first recurrence (Group A, 4.2 months; Group B, 4.1 months; $p>0.05)$. Seven of 17 (41\%) patients in Group $A$ and 16 of 25 (64\%) patients in Group B had disease progression and underwent radical cystectomy $(p<0.05)$. Both intravesical administrations were generally well tolerated. Conclusion: Thiotepa is a promising intravesical agent for treatment of non-muscle invasive bladder cancer.
\end{abstract}

Copyright $\odot 2012$ S. Karger AG, Basel
(C) 2012 S. Karger AG, Basel

1015-9770/12/0063-0160\$26.00/0

Fax +4161306 1234

E-Mail karger@karger.ch

www.karger.com

\section{Introduction}

Urothelial cancer of the bladder is the fourth most common malignancy diagnosed in American men [1]. The majority of these cancers are non-muscle invasive lesions at the time of diagnosis [2]. The use of intravesical chemotherapeutic agents in the management of superficial bladder cancer is based on the premise that an effective dose of a tumoricidal agent can be delivered directly to the tumor without significant adverse systemic effects. If we consider carcinoma of the bladder to be a field change disease, then another advantage of intravesical therapy is that it treats the abnormal epithelium elsewhere in the bladder as well as the localized tumor.

The initial therapy for patients with non-muscle invasive bladder carcinoma is generally transurethral resection and fulguration. The 5-year survival for these patients ranges from 63 to $82 \%$ [3]. However, the rate of recurrence and/or new tumor formation following local resection of these superficial bladder tumors is high, varying between 50 and 70\% [4-7].

Intravesical bacillus Calmette-Guérin (BCG) is so far the most effective and common form of adjuvant therapy for bladder cancer [8]. Compared with controls, BCG immunotherapy has superior advantage in preventing tumor recurrence over intravesical chemotherapy $[9,10]$. In contrast to intravesical chemotherapy, BCG has also been shown to reduce the risk of tumor progression [11]. Despite its success, significant proportions (30-40\%) of patients do not respond to BCG therapy and 30 to $50 \%$ of

Raheleh Sadat Sajadi Nia

Shahid Beheshti University of Medical Sciences, Velenjak Tehran (Iran)

E-Mail rahelehsajadinia@yahoo.com 
initial responders have a relapse within the first 5 years [12]. Furthermore, side-effects are common, which may be related to the BCG dose, especially in highly sensitive patients [13].

In 1961 and 1962, Jones et al. [14] and Veenema et al. [15] demonstrated that the instillation of thiotepa (triethylenethiophosphoramide) into the bladder destroyed low-stage bladder carcinoma in some patients. Since then there have been numerous reports regarding thiotepa both as a therapeutic agent for superficial bladder carcinoma and as a prophylactic agent [16-19]. These reports indicate that tumors will be destroyed in about one-third of the patients, diminished in size or number in another one-third, and unaltered in the remaining one-third. The effectiveness of this compound in eliminating existing tumors has been repeatedly demonstrated [19-22]. On the other hand, its role in the prevention of superficial recurrence remains controversial.

A randomized trial, in which thiotepa was compared with a placebo and pyridoxine, showed the lowest recurrence rate to be in the group treated with thiotepa, but the differences between the groups were not significant [23]. Thus, thiotepa has convincingly demonstrated a capacity to destroy tumors, but its ability to prevent recurrence is being increasingly questioned.

The aim of our study was to evaluate the efficacy of intravesical thiotepa compared with administration of $\mathrm{BCG}$ in the recurrence and progression of non muscle invasive bladder cancer.

\section{Materials and Methods}

This was a multicenter, prospective, randomized study carried out between June 2009 and May 2011. The study was approved by the local research ethical committee of each participating center. Written informed consent was obtained from all patients.

Of 72 patients with proven in situ bladder cancer, who were eligible and enrolled in this study, 66 were men and 6 were women. They ranged in age at the time of diagnosis from 31 to 87 years, with an average of 65.6 years (63.7 years for the men and 67.1 years for the women).

Symptoms brought 66 patients to the physician; of the 6 who were asymptomatic, all had micro-hematuria as a cause for the urological consultation. By far the most common symptoms were those of bladder irritation (dysuria, urgency, and frequency) in 60 patients. Three other patients complained of gross hematuria, and 3 had penile pain. The average duration of symptoms among the symptomatic group before the first cytological abnormality was detected was 22.2 months. Cystoscopic examination revealed no overt neoplasm in any patient. The most frequently noted cystoscopic abnormalities were increased vasculanity of the mucosa and erythematous granular zones without clearly defined margins.

Thiotepa versus BCG in Non-Muscle

Invasive Bladder Cancer
Tumors were pathologically staged according to the 1997 TNM classification. Pathological findings revealed that all the carcinomas were transitional, and in none was squamous (epidermoid) metaplasia noted. All showed moderate to severe degrees of anaplasia, Grades 3 and 4 on Broders' scale. Urine cytological findings generally consisted of large, anaplastic, mostly single cells. Decreased intercellular cohesiveness was evident in the sections of altered mucosa and accounted for both the abundant shedding of cells into the urine and the pronounced fragility of the mucosal surface, which often produced the artifact of mucosa missing from portions of the biopsy specimens.

\section{Study Design}

All patients started treatment after 4 to 6 weeks from the last transurethral resection (TUR), by using a central computer-generated randomization list; patients were randomly allocated to 1 of the 2 groups. An open-label study design was used, that is, patients and investigators were not masked as to the drugs they were assigned.

Group A received intravesical thiotepa, once weekly at a dose of $30 \mathrm{mg} / 30 \mathrm{ml}$ for 9 consecutive weeks (induction course), and then monthly for 12 months. Group B was given intravesical BCG (Connaught strain, $80 \mathrm{mg} / 50 \mathrm{ml}$ ) over a 9-week induction course and then each week for 3 weeks, at 3, 6, and 12 months.

The pre-study clinical evaluation comprised medical history, general physical examination, electrocardiogram, computed tomography (CT)-urography, chest x-ray, and hematological evaluation (including white blood cell-platelet count, electrolytes, and liver and kidney function). Urine analyses with urine culture were also done weekly during the treatment. Clinical, hematological, and biochemical assessments were performed every third week and were repeated at the end of treatment. Cytological analysis of voided urine and cystoscopy were performed at 3-month intervals. Intravenous urography or CT-urography was performed annually.

Recurrence was determined by lesions that were detected by cystoscopy and pathologically confirmed after TUR. A positive cytology alone was not considered as a recurrence. In case of positive cytology, a bladder mapping was performed, and only if there was pathological confirmation of a tumor was a case counted as a recurrence. Time to first recurrence was defined as the time from TUR to the date of the first recurrence.

Progression was defined as an increase in tumor stage and grade. Time to progression was defined as the time between TUR and first progression.

Toxicity was assessed on the first day of each cycle with the use of the Common Toxicity Criteria version 3.0.15, Grade 3 side effects resulted in patients' exclusion from the study. In the case of grade 2 toxicity, the treatment was delayed for 1 week and repeated. If toxicity relapsed at grade 2 , the treatment was stopped. Side effects were checked after each instillation and were recorded in the database. No dose reduction was allowed.

\section{Statistics}

The primary endpoint was the recurrence rate (percentage of recurring patients) at 1-year follow-up. Secondary endpoints were time to recurrence, progression rate, time to progression, and toxicity.

Quantitative data were described by the median (range), and qualitative data were described as counts and percentages. Chi- 
Table 1. Baseline patient characteristics in Group A $(n=36)$ and $\mathrm{B}(\mathrm{n}=36)$

\begin{tabular}{|c|c|c|c|}
\hline Characteristics & Group A & Group B & $\mathrm{p}$ \\
\hline Men/women & $34 / 2$ & $32 / 4$ & NS \\
\hline Mean age, years & 65.3 & 65.9 & NS \\
\hline Classification & & & NS \\
\hline $\mathrm{Ta}$ & 12 & 10 & \\
\hline $\mathrm{T} 1$ & 24 & 26 & \\
\hline Grade & & & NS \\
\hline 3 & 16 & 14 & \\
\hline 4 & 20 & 22 & \\
\hline Number of tumors & & & NS \\
\hline $2-8$ & 30 & 28 & \\
\hline$\geq 9$ & 6 & 8 & \\
\hline Tumor diameter & & & NS \\
\hline$<3 \mathrm{~cm}$ & 28 & 30 & \\
\hline$\geq 3 \mathrm{~cm}$ & 8 & 6 & \\
\hline
\end{tabular}

square and Fisher exact test were used to assess the significance of all correlations. Statistical significance was achieved if $\mathrm{p}<0.05$. All reported $p$ values were 2 -sided. All data were recorded, collected, and analyzed using standard statistical software.

\section{Results}

Of 92 initially screened patients with non-muscle invasive bladder cancer, 72 were eligible and enrolled in this study. All patients were randomly assigned to 2 groups of 36 (Groups A and B). The clinical and pathological characteristics of the 2 groups are shown in table 1 . Median follow-up was 15.2 months (range 6-22 months) in Group A and 15.8 months (range 7-21 months) in Group B.

\section{Disease Recurrence}

In Group A, 17 of 36 (47.2\%) patients developed disease recurrence versus 25 of $36(69.4 \%)$ in Group B (p $<0.05$ ). The difference between the 2 groups in terms of time to first recurrence (Group A: 4.2 months; 95\% confidence interval [CI]; Group B: 4.1 months; 95\% CI) was not statistically significant (hazard ratio 1.1; CI 95\%, $\mathrm{p}>0.05)$.

\section{Disease Progression}

Seven of 17 (41\%) patients in Group A and 16 of 25 $(64 \%)$ patients in Group B had disease progression $(\mathrm{p}<$ 0.05 ) and underwent radical cystectomy with ileostomy or ureterocutaneostomy. Moreover, 3 of 17 (17.6\%) pa-
Table 2. Toxicity in the treatment groups

Toxicity Group A Group B p

Dysuria

Grade 2

Grade 3

Hematuria

Grade 2

Grade 3

Fever

Grade 2

Grade 3

Neutropenia-thrombocytopenia

Grade 2

Grade 3

Dermatitis

Grade 2

Grade 3

Nausea-vomiting

Grade 2

Grade 3

Total events

Grade 2

Grade 3

$\begin{array}{rrr}3 & 7 & \\ 1 & 1 & \\ 2 & 4 & \\ 0 & 1 & \\ & & \\ 2 & 3 & \\ 0 & 1 & \\ & & \\ 1 & 0 & \\ 1 & 0 & \\ & & \\ 2 & 1 & \\ 0 & 0 & \\ & & \\ 2 & 0 & >0.05 \\ 1 & 0 & >0.05 \\ 12 & 15 & \end{array}$

tients in Group A and 6 of 25 (24\%) patients in Group B submitted to radiation therapy plus systemic chemotherapy.

At the time of the last follow-up visit, all patients were alive in Group A, and 1 had died because of metastatic disease in Group B. No statistically significant difference was reported $(\mathrm{p}>0.05)$.

\section{Toxicity}

Both intravesical administrations of thiotepa and BCG were generally well tolerated (table 2). Overall, few severe (grade 3 ) adverse events occurred, with no statistically significant difference between the 2 groups. We observed 1 case grade 3 dysuria, 1 case nausea-vomiting and 1 case grade 3 thrombocytopenia in Group A. One case of dysuria, 1 of hematuria, and 1 of fever $(>380 \mathrm{C})$ represented the grade 3 events in Group B. In all these 5 cases, treatment was delayed, accounting for a $10 \%$ delay rate in both groups.

\section{Discussion}

Transurethral resection is the initial treatment for patients with non-muscle invasive UCB. Unfortunately, 
these tumors recur in 40 to $80 \%$ of patients following TUR [24]. To prevent tumor recurrence and progression, intravesical instillation of BCG is the most commonly used adjuvant therapy. To date, there are limited proven effective alternative intravesical therapies for patients with BCG-refractory bladder cancers. Increasing the dose of BCG or enhancing the treatment schedules was suggested, but was associated with higher toxicities [25, 26].

The alkylating agents like thiotepa are the single most useful group of the cancer chemotherapeutic agents, even though they can not significantly prolong the life of patients in most malignancies. There has been much controversy as to whether basic differences exist between the various alkylating agents [27-30].

The absorptive properties of the bladder were studied by Maluf [31], who found that the bladder acts as a poorly permeable membrane, allowing the absorption of some chemical substances by a process of simple diffusion. Molecular size, therefore, determines absorption from the bladder and the substances absorbed have molecular weights of lower than 200. The molecular weight of thiotepa is 189 and it was therefore expected it would be absorbed.

Using a chemical method of estimation devised by Raine [32], it was shown that thiotepa was absorbed from the bladder. In 2 patients first treated with this technique, a small total dose of $30 \mathrm{mg}$ of thiotepa in $100 \mathrm{ml}$ sterile water was used, and the absorption of the drug was chemically estimated. It was found that one-third of the thiotepa had been absorbed and our future regime was based on the assumption that significant absorption of thiotepa would occur.

Previous investigations have shown that the alkylating agents produce their effect by interstrand binding from the N7 in guanine of one DNA strand to the N7 of guanine on the opposite strand [27-30]. The interstrand binding of the polyfunctional alkylating agents prevents the separation of the two strands of DNA in the double coiled helix necessary for cell replication, and thus inhibits cell proliferation in actively growing neoplastic cells. Alkylating agents including thiotepa have been reported to keep concentration dependent cytotoxicity against proliferating cells in general [33-35].

Jones et al. [14] and Veenema et al. [15] first reported on the antitumor activity of thiotepa instilled into the bladder nearly 20 years ago. Early reports attributed a prophylactic value to the drug [36] and this was confirmed in a controlled trial employing a single dose of thiotepa [37]. In a more recent controlled study the drug was found to be ineffective in lowering the recurrence rate [20]. The European Organization for Research on the Treatment of Cancer [38] investigated the effectiveness of thiotepa and VM-26 (a semisynthetic podophyllotoxin derivative) in the prevention of recurrence of superficial bladder cancer. In their trial 115 patients received thiotepa and 116 VM-26 and 109 served as controls. Although the recurrence rate was lower in the group receiving thiotepa, the differences between the 3 groups were not significant.

To our knowledge, our study is the first prospective randomized study to compare intravesical thiotepa to BCG in this selected subset of patients with non-muscleinvasive bladder cancer.

We found thiotepa to be more effective than BCG in reducing recurrence rates $(47.2$ vs. $69.4 \%, \mathrm{p}<0.05)$, whereas no significant difference was found in terms of time to first recurrence (4.2 vs. 4.1 months in Group B, p $>0.05)$. Furthermore, disease progression was lower in Group A (41 vs. $64 \%, \mathrm{p}<0.05$ ). Moreover, $17.6 \%$ of patients in Group A and 24\% in Group B needed systemic chemotherapy and/or radiation.

Thiotepa was administered with an extensive schedule (once weekly for 9 weeks). Of course, it is clear that no standard regimen exists in this setting, and the optimal frequency and duration of maintenance instillations remain unknown. Thus, further investigation addressing this issue is needed.

The major concern was obviously related to the potential toxicity. Urinary symptoms represented the main adverse events in both study groups. They were mostly managed successfully with anticholinergic, antibiotic, and/or anti-inflammatory drugs. Treatment-specific side effects, such as nausea, dermatitis, and thrombocytopenia, were unique to the gemcitabine-treated group, whereas hematuria and cystitis appeared more frequently in the BCG group. Overall, our data support the use of such an intensive schedule in terms of toxicity profile, because intravesical administration of thiotepa was generally well tolerated.

Based on our study, thiotepa showed higher efficacy in limiting the recurrence and progression of non muscle invasive bladder cancer and therefore is a promising intravesical agent for treatment of non muscle invasive bladder cancer. This alkylating agent warrants further exploration in patients with non muscle invasive bladder cancer, especially in those with BCG refractory diseases. 


\section{Acknowledgments}

The authors thank The Microbiology Department of Shahid Beheshti Medical University, Dr. Reza Fahimi for his general support and all Urology Departments of the hospitals affiliated with Shahid Beheshti University of Medical Sciences.

\section{References}

1 Jemal A, Siegel R, Xu J, Ward E: Cancer sta- 15 Veenema RJ, Dean AL Jr, Roberts M, Fingtistics, 2010. CA Cancer J Clin 2010;60:277300.

2 Xiao Z, Hanel E, Mak A, Moore RB: Antitumor efficacy of intravesical BCG, Gemcitabine, Interferon- $\alpha$ and Interleukin-2 as mono- or combination-therapy for bladder cancer in an orthotopic tumor model. Clin Med Insights Oncol 2011;5:315-323.

-3 Prout GR Jr: The surgical management of bladder carcinoma. Urol Clin North Am 1976;3:149-175.

4 Franksson C: Tumours of the urinary bladder: a pathological and clinical study of 434 Cases. Acta Chir Scand 1950;100:664-667.

$\checkmark 5$ Greene LF, Hanash KA, Farrow GM: Benign papilloma or papillary carcinoma of the bladder. J Urol 1973;110:205-207.

6 Greene LF, Yalowitz PA: The advisability of concomitant transurethral excision of vesical neoplasm and prostatic hyperplasia. J Urol 1972;107:445-447.

7 Nichols JA, Marshall VF: Treatment of histologically benign papilloma of the urinary bladder by local excision and fulgeration. Cancer 1956;9:566-567.

-8 Herr HW, Morales A: History of bacillus Calmette-Guérin and bladder cancer: an immunotherapy success story. J Urol 2008; 179: 53-56.

99 Lamm DL: Bacillus Calmette-Guérin immunotherapy for bladder cancer. J Urol 1985; 134:40-47.

$\checkmark 10$ Herr HW, Warringer DD, Fair WR, Oettgen HF: Bacillus Calmette-Guerin therapy for superficial bladder cancer: a 10 year followup. J Urol 1992;147:1020-1023.

11 Amling CL: Diagnosis and management of superficial bladder cancer. Curr Probl Cancer 2001;25:217-278.

12 Lamm DL, Griffith JG: Intravesical therapy: does it affect the natural history of superficial bladder cancer? Semin Urol 1992;10:39-44.

13 Lamm DL: Complications of Bacillus Calmette-Guerin immunotherapy. Urol Clin North Am 1992;19:565-572.

14 Jones HC, Swinney J: Thiotepa in the treatment of tumours of the bladder. Lancet 1961; 2:615-618. erhut B, Chowhury BK, Tarassoly H: Bladder carcinoma treated by direct instillation of thio-TEPA. J Urol 1962;88:60-63.

16 Abbassian A, Wallace DM: Intracavitary chemotherapy of diffuse non-infiltrating papillary carcinoma of the bladder. J Urol 1966; 96:461-465.

17 Edsmyr F, Boman J: Instillation of thio-tepa (Tifosyl) in vesical papillomatosis. Acta Radiol Ther Phys Biol 1970;9:395-400.

18 Pavone-Macaluso M: Chemotherapy of vesical and prostatic tumours. Br J Urol 1971;43: 701-708.

19 Veenema AJ, Dean AL Jr, Usom AC, Roberts M, Longo F: Thiotepa bladder instillations: therapy and prophylaxis for superficial bladder tumors. J Urol 1969;101:711-715.

20 Nocks BN, Nieh PT, Prout GR Jr: A longitudinal study of patients with superficial bladder carcinoma successfully treated with weekly intravesical thio-tepa. J Urol 1979;122:27.

-21 National Bladder Cancer Collaborative Group A: The role of intravesical thiotepa in the management of superficial bladder cancer. Cancer Res 1977;37:2916-2917.

22 Edsmyr F, Boman J: Instillation of thio-tepa (Tifosyl) in vesical papillomatosis. Acta Radiol Ther Phys Biol 1970;9:395-400.

-23 Byar D, Blackard C: Comparisons of placebo, pyridoxine, and topical thiotepa in preventing recurrence of stage I bladder cancer. Urology 1977;10:556-561.

-24 Soloway MS, Sofer M, Vaidya A: Contemporary management of stage $\mathrm{T} 1$ transitional cell carcinoma of the bladder. J Urol 2002;167: 1573-1583.

25 Lamm DL: Complications of Bacillus Calmette-Guerin immunotherapy. Urol Clin North Am 1992;19:565-572.

-26 Grossman HB, O'Donnell MA, Cookson MS, Greenberg RE, Keane TE: Bacillus CalmetteGuerin failures and beyond: contemporary management of non-muscle-invasive bladder cancer. Rev Urol 2008;10:281-289.
27 Pullman B, Pullman A: The electronic structure of the purine-pyrimidine pairs of DNA. Biochim Biophys Acta 1959;36:343-350.

28 Brookes P: Reaction of alkylating agents with nucleic acid; in Plattner PA (ed): Chemotherapy of cancer. Elsevier Publishing Company, Amsterdam, London \& New York, 1964, pp32-43.

29 Lawley PD, Brookes P: Molecular mechanism of the cytotoxic action of difunctional alkylating agents and of resistance to this action. Nature 1965;206:480-483

30 Roberts JJ, Warwick GP: Studies of the mode of action of tumour-growth-inhibiting alkylating agents. IV. In vitro reactions of 2chloroethylarylamines. Biochem. Pharmacol 1963;12:1315-1319.

-31 Maluf NS: Absorption of water, urea, glucose and electrolytes through the human bladder. J Urol 1953;69:396-404.

32 Raine DN: Estimation of thiotepa in urine. J Pharm Pharmacol 1962;14:614-615.

33 Shimoyama M: Implication of kinetics of cell killing by anticancer agents in the design of optimal therapeutic schedules. Saishin-Igaku (Jap) 1973;28:850-859.

34 van Putten LM: Are cell kinetic data relevant for the design of tumour chemotherapy schedules? Cell Tissue Kinet 1974:7:493-504.

35 Freshney RI, Paul J, Kane IM: Assay of anticancer drugs in tissue culture; conditions affecting their ability to incorporate $3 \mathrm{H}$-leucine after drug treatment. Br J Cancer 1975;31: 89-99.

36 Drew JE, Marshall VF: The effects of topical thiotepa on the recurrence rate of superficial bladder cancers. J Urol 1968;99:740-743.

37 Collins WE: TNM classification of malignant tumours of the bladder, prostate, testis and kidney. Can J Surg 1975;18:468-475.

38 European Organization for Research on the Treatment of Cancer: Chemotherapy in superficial bladder cancer; in Oliver $\mathrm{T}$ (ed): New approaches in the treatment of bladder cancer. Butterworths, London, 1980. 